\title{
O FARMACEUTICO NO CONTEXTO DA ESTRATÉGIA EM SAÚDE DA FAMÍLIA, QUE REALIDADE É ESTA?
}

\section{THE PHARMACIST IN THE CONTEXT OF FAMILY HEALTH STRATEGY, WHICH REALITY IS THIS?}

\author{
RICIERI, M.C. ${ }^{1 \star}$, PREVIATTI, D. ${ }^{1}$, CAMPESE, M. ${ }^{1}$, CONSTANTINI, H. F. ${ }^{2}$, \\ MONTRUCCHIO, D.P. ${ }^{3}$ KADES, A.S.O. ${ }^{4}$, BUFFON, M.C.M. ${ }^{5}$, FURMAN, I.M. ${ }^{6}$ \\ 1. Farmacêuticos Residentes do Programa de Residência Multiprofissional em Saúde da Família da \\ UFPR; \\ 2. Farmacêutica Preceptora do Programa de Residência Multiprofissional em Saúde da Família da UFPR; \\ 3. Farmacêutica Professora Supervisora do Programa de Residência Multiprofissional em Saúde da \\ Família da UFPR; \\ 4. Nutricionista Residente do Programa de Residência Multiprofissional em Saúde da Família da UFPR; \\ 5. Cirurgiã Dentista Professora Supervisora do Programa de Residência Multiprofissional em Saúde da \\ Família da UFPR; \\ 6. Médica da Família da Unidade de Saúde São Domingos, Município de Colombo. \\ * marisricci@ufpr.br
}

\section{RESUMO}

O presente artigo relata a experiência do profissional farmacêutico no âmbito da Atenção Primária, no contexto da Estratégia de Saúde da Família (ESF). Resgata seu histórico até a sua inserção em uma unidade de saúde, discute onde se insere o farmacêutico na proposta de transitoriedade do perfil da assistência e as possíveis abordagens dentro do campo da saúde coletiva. Aponta as dificuldades encontradas durante o período de residência em Unidades de Saúde da Família (USF), as necessidades na formação desse profissional para atuar na Atenção Primária à Saúde e, as diferentes configurações que o medicamento assume perante o indivíduo, bem como o trabalho integrado à equipe multiprofissional, em especial ao profissional médico e ao nutricionista.

Palavras chave: farmacêutico, estratégia saúde da família, unidade de saúde.

\section{ABSTRACT}

The present article reports the pharmacist's experience on primary health attention, in the context of Brazilian family health strategy. It shows the path to his insertion in the health units, discusses where and how this pharmacist can act in this new proposal and the possible approaches on collective health field. It also brings a reflection about the difficulties during the period of Residence on health family units, the needs of professional education and the different meanings of the medicine to the patient, as well as the integrated work with the multiprofessional team, specially medicine and nutrition. Keywords: pharmacist; family health strategy; health unit. 


\section{PERCURSO HISTÓRICO DA PROFISSÃO FARMACÊUTICA}

A profissão farmacêutica é uma das profissões mais antigas da história. E ao longo de sua trajetória passou por crises, mudanças e evolução, transpondo momentos históricos importantes, determinados pelos diferentes cenários políticos, econômicos e sociais.

O primeiro momento refere-se à figura do farmacêutico associado ao profissional elaborador do medicamento - o boticário. Nessa época, a função era seguir, essencialmente, a pesquisa e manipulação de formas extemporâneas. As boticas (farmácias) mantinham a hegemonia da preparação de medicamentos receitados pelos médicos, sendo noventa por cento do trabalho do farmacêutico efetuado no laboratório farmacotécnico, desenvolvendo as fórmulas magistrais. Nesse reduto, pairava o prestígio e a influência do farmacêutico (PERETTA; CICCIA, 2000; HEPLER; STRAND, 1999).

No Brasil, a industrialização do medicamento, iniciada a partir de 1920, a abertura da economia ao capital estrangeiro e a instalação das indústrias farmacêuticas transnacionais no país, levaram ao desaparecimento das boticas. O farmacêutico foi convertendo-se em um mero intermediário entre a indústria e o usuário (PERETTA; CICCIA, 2000).

Essas indústrias, por funcionarem sob a lógica do lucro e curativa da doença, levaram à descaracterização do farmacêutico como profissional de saúde, passando a ser referenciado como o profissional do medicamento, por trabalhar somente questões relativas à produção. Em função disso, instalou-se a chamada "crise de identidade" do farmacêutico, agravada pelas lacunas da formação e pelo não reconhecimento social da profissão (LAPORTE, 1989).

Foi nesse contexto, em meados da década de 60, que houve um desdobramento da profissão farmacêutica para o campo das análises clínicas, conferindo, ao farmacêutico, habilidades para procedimentos prioritariamente técnicos. 
Dessa forma, pode-se afirmar que os campos de atuação do farmacêutico na indústria, nas análises clínicas, no hospital e nas farmácias de dispensação e manipulação são campos da farmácia que já estão bem estabelecidos e consolidados.

Porém, evidencia-se que o trajeto percorrido pela profissão promoveu um distanciamento do farmacêutico em relação ao indivíduo e à coletividade, o que levou o farmacêutico a repensar práticas e medidas, visando maior aproximação com o usuário.

Essa intenção foi possível através do sistema de saúde vigente no Brasil - o Sistema Único de Saúde (SUS) -, advindo do Movimento da Reforma Sanitária que tem como princípios básicos a universalidade, a igualdade ${ }^{1}$ e a integralidade (BRASIL, 2006). Este último princípio visa prestar ao indivíduo atenção integral, sob os aspectos da prevenção de doenças e promoção e recuperação da saúde, e foi o que inspirou a conquista de mais um campo da Farmácia, o da Atenção Primária, tendo como espaço de atuação, a Unidade de Saúde.

Nessa perspectiva, os diferentes contextos históricos que passamos exigiram do farmacêutico dinamismo na sua atuação. A sociedade necessita de outras abordagens e o campo da saúde coletiva pode oferecer várias ferramentas para atuações mais efetivas e de qualidade.

\section{O FARMACÊUTICO NA ESTRATÉGIA EM SAÚDE DA FAMÍLIA}

Todos nós sabemos dos inquestionáveis progressos da medicina ocidental. Porém, a organização do sistema médico e a estrutura das sociedades industriais, na qual nos incluímos, apresentam importantes problemas como, por exemplo, o crescimento exponencial dos gastos em saúde, as enfermidades desencadeadas pelo modo de produção industrial/condições de trabalho e a medicalização da sociedade (POLIGNANO, 200-; VELAZQUEZ, 1986).

Para entendermos o contexto da Estratégia em Saúde da Família (ESF), tendo sua práxis desenvolvida no território da Unidade de Saúde, nos remetemos ao modelo

\footnotetext{
${ }^{1}$ Muitos autores utilizam como princípio do SUS a equidade, que é diferente de igualdade. A equidade é um princípio de justiça social, ou seja, a rede de serviços deve atender às necessidades da população que mais precisa da assistência. Porém, os documentos oficiais que regulamentam o SUS, como a Constituição Federal e a Lei n. 8.080/90 referem-se a igualdade (POLIGNANO, 200-).
} 
de atenção vigente no Brasil, o chamado modelo biomédico, e a contrapartida da ESF frente a esse modelo e onde se insere o farmacêutico nessa proposta de transitoriedade do perfil da assistência.

A lógica do modelo biomédico, segundo CAPRA (1982) e MERHY et al (2004), é reducionista e individualista, ou seja, o homem é tratado como uma máquina composta de várias peças e a função da medicina é manter o bom estado de funcionamento do corpo/máquina, para que o homem não perca seu posto nas relações de produção. É também um modelo hospitalocêntrico e médico-centrado, isto é, infunde-se a cultura de que é somente no hospital que os problemas são resolvidos e que o único profissional que pode "curar" (quando acredita-se na perspectiva da cura) é o médico. Logo, esse modelo segue sendo curativista e medicamentoso e, por conseguinte, oneroso.

O farmacêutico, na lógica desse modelo, insere-se como o profissional que analisa a prescrição, detendo-se às questões implícitas na receita médica. Ou em outros setores, como na indústria, é responsável pela produção de fármacos e, em hospitais, responsabiliza-se, essencialmente, pelo gerenciamento de medicamentos e questões administrativas.

A ESF apresenta-se como o recurso mais apropriado para a reorganização das práticas da Atenção Básica e reversão do atual modelo de assistência, tendo como proposta trabalhar com os princípios e diretrizes do SUS. As características gerais da ESF estão pautadas no binômio epidemiologia/vigilância à saúde, em substituição às práticas convencionais de assistência; no trabalho com uma base territorial definida; e com o cadastro e acompanhamento da população adscrita da área (BRASIL, 1997; MERHY et al, 2004).

Adotando a ESF como um novo norte para a assistência, vivenciamos o embate do modelo de atenção deixar de ser médico-centrado para instituir a participação e responsabilização de outros profissionais, que procuram trabalhar de forma multiprofissional e interdisciplinar. Nesse horizonte, a busca da intersetorialidade é estratégica para a Saúde da Família, partindo da premissa que o processo saúdedoença compõe-se, dentre outros fatores, de determinantes sociais. 
Em linhas gerais, a lógica da ESF volta-se para a promoção e o conceito ampliado de saúde. Todo esse redirecionamento tem a prioridade de diminuir custos, pois se pensa que o enfoque epidemiológico, aliado à abordagem clínica, na Atenção Primária, pode ser responsável pela resolubilidade de 85\% dos problemas de saúde mais comuns (SCHERER et al, 2005; MERHY et al, 2004).

Nesse contexto da Estratégia em Saúde da Família, é factível ao farmacêutico muito mais dinamismo nas suas ações, abrindo espaço para atuações desvinculadas diretamente da prescrição médica. O farmacêutico deixa a posição anquilosada posta no modelo biomédico - centrado no medicamento - e volta-se para o trabalho integrado à equipe multiprofissional, à coletividade, à sociedade e ao sujeito, numa perspectiva sistêmica.

Logo, o primeiro questionamento que trazemos é se o farmacêutico está preparado para transpor o modelo biomédico hegemônico e atuar no processo de construção do SUS.

\section{O FARMACÊUTICO NA UNIDADE DE SAÚDE DA FAMÍLIA - UM RELATO DE EXPERIÊNCIA}

Como um dos objetivos desse artigo é relatar a experiência do farmacêutico no universo da Atenção Primária, desenvolvendo suas práticas na Unidade de Saúde da Família (USF), iniciamos esclarecendo que a inserção do farmacêutico nesse campo de atuação ocorreu devido ao Programa de Residência Multiprofissional em Saúde da Família (PRMSF) da Universidade Federal de Paraná, projeto que propõe novas relações de ensino/formação/serviço, as quais vem sendo incentivadas pela política de Educação Permanente em Saúde.

O PRMSF se desenvolve sob dois eixos: a fundamentação teórica (aulas semanais, com carga horária total 828 horas) e a prática em serviço (com carga horária de 4692h) realizada nas Unidades de Saúde da Família, no Município de Colombo, localizado na região Metropolitana de Curitiba, com aproximadamente 230.000 habitantes. 
O projeto da Residência busca priorizar a educação dos profissionais de saúde e impele a transformações do trabalho no setor saúde, para que o serviço e o processo de trabalho seja o lugar de apropriação de conhecimentos, atuação crítica, reflexiva, propositiva e tecnicamente competente.

Na nossa atuação como farmacêuticos residentes, desde os primeiros meses na Residência, a realidade encontrada nas USF não favorecia a prática farmacêutica por vários motivos. O primeiro deles está relacionado com a Política Nacional de Medicamentos, que visa à reorientação da Assistência Farmacêutica, a fim de garantir a necessária segurança, eficácia e qualidade dos medicamentos, a promoção do seu uso racional e o acesso da população àqueles medicamentos considerados essenciais. Para isso, adota algumas diretrizes, entre elas, a Assistência Farmacêutica na Atenção Primária, entendida como parte importante do sistema de saúde e componente necessário para a efetivação dos princípios dos SUS (BRASIL, 2001).

A Política de Assistência Farmacêutica, desenvolvida operacionalmente no ciclo da Assistência Farmacêutica (BRASIL, 2001), ainda não permeia, em todas as suas etapas e no âmbito da Atenção Primária, a presença do farmacêutico. Logo, a chegada e a inserção desse profissional foram dificultadas pela falta de referencial do farmacêutico no serviço. Não existe um papel definido das competências e limites da atuação farmacêutica, porque ainda são pouco difundidas e incipientes as experiências do farmacêutico na Atenção Primária e na ESF.

A expectativa é que agora com a promulgação da Portaria 698/06, a qual prevê recursos financeiros federais para os serviços farmacêuticos na Atenção Primária, os Municípios sintam-se incentivados a contratarem farmacêuticos para atuarem, inclusive nas USF (BRANDÃO, 2006). Essa proposta certamente conduzirá a profissão farmacêutica a construir referenciais de prática no âmbito da Atenção Primária.

O segundo aspecto pontual é que, embora a dinâmica das Unidades de Saúde ainda esteja centrada no atendimento médico, nos programas de saúde e nos medicamentos, não existe espaço físico próprio para a Farmácia na maior parte das USF. Os medicamentos são armazenados em armários localizados, geralmente, na sala da coordenação da unidade, o que prejudica as ações que visam à manutenção da 
qualidade dos medicamentos e o seu uso racional, como o controle de estoque, o armazenamento e a dispensação. A distribuição ou entrega dos medicamentos é realizada por qualquer profissional da equipe, não havendo ações sistemáticas referentes ao controle de estoque, validade e consumo médio mensal, o que repercute em um precário gerenciamento, incidindo em custos para o Município.

Os medicamentos da Farmácia Básica (Relação Municipal de Medicamentos Essenciais) são concentrados em algumas farmácias satélites do Município e, as US em que estivemos alocados, só dispunham de medicamentos vinculados aos programas Hiperdia (Plano de Reorganização da Atenção à Hipertensão Arterial e ao Diabetes Mellitus) e Planejamento Familiar, além daqueles contidos no kit PSF (medicamentos padronizados pelo Ministério da Saúde). Esse kit era distribuído sem uma periodicidade definida e com alguns itens que não eram necessários naquela população, por não respeitarem os principais agravos de saúde a nível local. Logo, essa restrição de medicamentos tornou-se um desafio para a realização e organização da Assistência Farmacêutica.

Somados a isso, está a grande proporção da população adscrita que faz uso de algum tipo de medicamento, principalmente para hipertensão, diabetes e saúde mental, problemas de saúde para as quais existem programas subsidiados pelo governo para acompanhamento e controle. O quadro dessa população, em geral, é de pessoas desinformadas quanto ao uso correto dos medicamentos, com dificuldades de acesso aos serviços de saúde e culturalmente adeptas do modelo medicalizado. Portanto, é imperioso afirmar a imprescindibilidade dos serviços farmacêuticos na Saúde Coletiva e, mais especificamente, na Atenção Primária à Saúde.

Em linhas gerais, essa foi a realidade - nada favorável - que nós, farmacêuticos residentes em Saúde da Família nos deparamos inicialmente. E diante disso, ponderamos: por onde começar nossa atuação?

Pensamos que para podermos nos inserir numa prática, devemos entender como operam as questões organizativo-estruturais que regem tal prática. Logo, a busca de conhecimentos sobre o SUS, ESF, processo de trabalho, equipe multiprofissional e 
questões ligadas ao medicamento na USF são requisitos iniciais para a construção das práticas em saúde do farmacêutico.

E essas práticas não poderiam, em momento algum, estar desconexas com a realidade da população. Realidade esta em que permeiam várias morbidades, diferentes conformações familiares, além de complexas questões sociais, políticas, econômicas e culturais e da dificuldade de acesso aos serviços de saúde.

Invariavelmente, em muitas das situações adversas vivenciadas na população, o medicamento apresentava-se atrelado de alguma maneira. Mas não de uma forma simplista, como um bem de consumo produzido industrialmente que age sobre determinada patologia, através de um mecanismo de ação. Para SEVALHO (2003), o medicamento industrializado deve ser considerado nas suas perspectivas de instrumento terapêutico e de bem de consumo, caracterizando-se como objeto científico híbrido. Entendendo que é elemento da complexidade social - ressaltando a sua caracterização como bem de consumo-, torna-se possível compreender de maneira crítica o conceito de uso racional do medicamento.

Portanto, o medicamento pode assumir várias facetas e valores simbólicos. Como comumente vivenciamos, ele pode ser referido como a "salvação dos problemas", como um símbolo de cura, como um aparato de dependência, ou como uma mercadoria ou um artifício que isola e camufla a realidade do indivíduo (LEFÈVRE, 1987).

Diante disso, outras provocações que surgem são, primeiro, se o farmacêutico pode ficar alheio à realidade e dinâmica da comunidade, considerando a riqueza e diversidade cultural; e segundo, se as questões ligadas ao medicamento podem ser tratadas de forma reducionista, sem levar em conta todas as configurações que o medicamento assume.

Em linhas gerais, considerar o contexto, o lugar onde vive o sujeito e o medicamento com seu valor simbólico requer que abandonemos o jargão do farmacêutico somente como profissional do medicamento e levantemos a bandeira do farmacêutico enquanto profissional de saúde, que para estabelecer relações de vínculo 
com o usuário e outros profissionais não precisa ter, necessariamente, o medicamento como ponto em comum.

Talvez a nova revolução da Farmácia aconteça quando o usuário estiver próximo ao farmacêutico (e vice-versa), vendo-o como um profissional que pode prestar uma assistência de qualidade. No entanto, para pôr em prática uma abordagem ampliada e segura como essa precisamos adquirir conhecimentos e habilidades de outras ordens, diferentes dos que a academia nos proporciona durante a graduação, haja visto o quanto somos limitados, em função da formação extremamente tecnicista do curso de Farmácia.

O fato de nos distanciarmos da assistência voltada exclusivamente para o medicamento, nos aproxima, ao mesmo tempo, de pensarmos no indivíduo como uma composição de vários microcontextos, formando uma rede sistêmica e indissociável, em que são relevantes aspectos como, por exemplo, considerar o indivíduo como membro de uma família, que assume um papel social e estabelece relações de poder; que tem sua cultura e suas crenças; de como este sujeito pensa a sua saúde; que ele pode ser (ou não) usuário de medicamento; e quais são as suas condições de trabalho e escolaridade. Enfim, são essas situações, entre outras, que são determinantes do processo saúde-doença de qualquer sujeito, difíceis de serem abordadas, em função do nosso despreparo, do modelo assistencial de saúde vigente e da própria cultura da população.

\section{PONTOS POSITIVOS DA ESF PARA A PRÁTICA FARMACÊUTICA}

A inserção do Farmacêutico na atenção primária, atuando na lógica da ESF, está diretamente vinculada ao novo perfil do profissional egresso disposto nas Diretrizes Nacionais Curriculares para Farmácia, as quais colocam que o farmacêutico deve estar preparado para atuar em todos os níveis de atenção à saúde de forma generalista, humanista, crítica e reflexiva "pautado em princípios éticos e na compreensão da realidade social, cultural e econômica do seu meio, dirigindo sua atuação para a 
transformação da realidade em beneficio da sociedade" (MEC/CNE/CES, Resolução no 02/02, Art. $3^{\circ}$ ).

Embora em meio a muitas dificuldades vivenciadas, relatamos que o cenário da ESF nos proporcionou pontos positivos que deram condições de manejo, efetividade e sustentabilidade para a nossa prática.

Um deles refere-se ao trabalho no campo, no território da USF, o qual expõe o farmacêutico a situações de exercício das suas competências e habilidades requeridas pela sociedade. Estamos falando do processo de territorialização, que identifica as características sociais, culturais, econômicas, perfil de morbi-mortalidade e definição de áreas e situações de risco.

O modelo de assistência a que se propõe a ESF milita a favor de uma assistência integral ao indivíduo. Espera-se, portanto, que o farmacêutico siga esse movimento, utilizando-se de peculiaridades que a estratégia dispõe como as visitas domiciliares, das quais os Agentes Comunitários de Saúde podem ser importantes mediadores para a atenção integral à saúde, a equipe multiprofissional, sistemas de coleta de dados, além de ferramentas como genograma, linha do tempo e ciclo de vida.

Com relação ao trabalho do farmacêutico dentro de uma proposta de trabalho multiprofissional, entendida aqui como uma integração de trabalhos caracterizada pela articulação das ações, interação entre os agentes e superação do isolamento dos saberes (PEDUZZI, 2001), merece destaque relatar, como foi o farmacêutico - com um perfil de trabalho individual e tecnicista -, inserir-se em uma equipe e desenvolver a multi e a interdisciplinaridade.

$\mathrm{Na}$ equipe de residentes estavam presentes as áreas da enfermagem, nutrição e odontologia, além da farmácia. O que ficou evidenciado na prática foi uma aproximação consistente entre a Nutrição e a Farmácia. Um dos motivos foi que esses dois campos não possuem profissionais estabelecidos na assistência local. Logo, as dificuldades e os anseios de conquistar espaço foram semelhantes.

O relato que a Nutricionista, de uma das equipes de residentes, fez acerca dessa busca pela integralidade dos saberes e vivências, foi de ter sido uma experiência muito construtiva, porque ao mesmo tempo em que trocávamos informações técnicas, 
podíamos estender nossa abordagem incluindo a percepção de outro profissional. Era um outro olhar presente, que muitas vezes não era técnico e sim, essencialmente subjetivo.

Outro fator enriquecedor do trabalho integrado é privilegiar um espaço para discussão na equipe. Isso acrescentou tanto para a tomada de decisões mais efetivas junto ao usuário/família, como para a construção do conhecimento a partir da experiência do outro, seja ele como técnico ou sujeito.

Vale ressaltar também que um outro ponto positivo da atuação do farmacêutico na Unidade de Saúde é a oportunidade de se trabalhar junto com o médico. A ESF foi um espaço privilegiado de relação, integração e cooperação entre esses dois profissionais, onde foi possível, entre outras coisas, discutir o processo da residência, condutas de tratamento, sanar dúvidas imediatas com relação à farmacoterapia e ao indivíduo/família e, principalmente, ter problematizado e refletido juntos sobre aspectos subjetivos que permanentemente envolvem as relações interpessoais e o processo saúde-doença.

Foi possível dividir com o profissional médico uma proposta de assistência baseada no princípio da integralidade e no conceito ampliado de saúde. Para o médico, ampliar a linha de cuidado ao usuário incluindo a abordagem de outros profissionais, foi uma iniciativa em que não houve resistências, pelo contrário, esteve repleta de incentivo e confiança.

\section{CONSIDERAÇÕES FINAIS}

Finalizando esse relato de experiência algumas considerações que deixamos é que as vantagens dessa estrutura proporcionada pela ESF, mesmo que de uma forma ainda deficiente e em processo de construção, tende a buscar e oferecer uma assistência de qualidade ao indivíduo, à família e à sociedade, garantindo-lhes os direitos que lhes foram legalizados.

$\mathrm{Na}$ condição de farmacêuticos residentes em Saúde da Família, ter vivido por dois anos essa experiência nos oportunizou conhecer a realidade da saúde no Brasil, 
conhecer a nós mesmos, lidar com situações adversas, sair do nosso tecnicismo e individualismo profissional e aprender a sermos, de fato, profissionais de saúde, úteis para a sociedade, dentro de preceitos éticos e humanitários.

\section{REFERÊNCIA}

BERMUDEZ, J. A. Z. Indústria Farmacêutica, Estado e Sociedade. Crítica da Política de Medicamentos no Brasil. São Paulo: HUCITEC/SOBRAVIME, 1995. 204 p.

BRANDÃO. A. O Farmacêutico na Atenção Básica. Pharmacia Brasileira, Brasília, n. 52, p. 4-10, mar./abr. 2006.

BRASIL. Ministério da Saúde. Secretaria de Assistência à Saúde. Coordenação de Saúde da Comunidade. Saúde da Família: uma estratégia para reorientação do modelo assistencial. Brasília: Ministério da Saúde, 1997. 36p.

BRASIL. Ministério da Educação. Conselho Nacional de Educação. Câmara de Educação Superior. Resolução CNE/CES n 2, de 19 de Fevereiro De 2002. Brasília, DF, 2002. Disponível em: <http://www.mec.gov.br/cne/pdf/CES022002.pdf> Acesso em: 25 jan. 2005.

BRASIL. Ministério da Saúde. Conselho Nacional de Saúde. Coletânea de Normas para o Controle Social no Sistema Único de Saúde. 2 ed. Brasília: Editora do Ministério da Saúde, 2006. 208 p.

CAPRA, F. O Ponto de Mutação. A Ciência, a Sociedade e a Cultura Emergente. São Paulo: Cultrix, 1982.

HEPLER, C.D.; STRAND, L.M. Oportunidades $Y$ responsabilidades en la Atención Farmacêutica. Pharm Care Esp., Madrid, v.1, v.1, p. 35-47, 1999.

LAPORTE, J.R. et al. Epidemiologia do Medicamento: princípios gerais. São Paulo/Rio de Janeiro: Hucitec-Abrasco, 1989.

LEFÈVRE, F. A oferta e a procura de saúde através do medicamento: proposta de um campo de pesquisa. Rev. Saúde Pública. Vol.21 no.1 São Paulo Feb. 1987.

MERHY, E.E. et al. O trabalho em saude: olhando e experienciando o SUS no cotidiano. 2 ed. São Paulo: Hucitec, 2004. p.55-124. 
PEDUZZI, M. Equipe Multiprofissional em Saúde: conceito e tipologia. Revista de Saúde Pública. 35(1):103-9. 2001

PERETTA, M. CICCIA, G. Reengenharia da Atenção Farmacêutica. Brasília: Ethosfarma, 2000.

POLIGNANO, M. V. História das Políticas de Saúde no Brasil: uma pequena revisão (200-). Disponível em: http://www.ead.fiocruz.br/facilitadores/ Acesso em: 12 out. 2006.

SCHERER, M.D.A. et al. Rupturas e resoluções de atenção à saúde: reflexões sobre a estratégia de saúde da família com base nas categorias kuhnianas. Interface. v.9, n.16, p.53-66, set.2004/fev.2005.

SEVALHO, G. in ACURCIO, F. A. Medicamentos e Assistência Farmacêutica. Belo Horizonte: Coopmed, 2003. Cap.1. p.1-8.

VELAZQUEZ, G. Salud, medicamentos y tercer mundo. Madrid: Iapala, 1986. 